\title{
Contribuciones de las experiencias de aprendizaje-servicio desde la perspectiva de estudiantes de la Universidad Alberto Hurtado
}

\section{Manuel Caire}

Universidad Alberto Hurtado, Chile

\section{Resumen}

Cuando se habla de aprendizaje servicio, se hace referencia a una propuesta pedagógica que busca desarrollar en los estudiantes aprendizajes intencionadamente planificados desde una asignatura, así como desarrollar un servicio que atienda necesidades reales de una comunidad. Se nutre de corrientes pedagógicas como el aprendizaje experiencial y el construccionismo. Sin embargo, la naturaleza de esta propuesta hace pensar que posee características de una intervención social. El objetivo del presente trabajo es describir la evaluación que los estudiantes de la UAH hacen sobre el aporte del aprendizaje servicio, tanto para su formación como para las organizaciones sociales. Se trató de un estudio exploratorio descriptivo con metodología mixta, utilizando un cuestionario semiestructurado con modalidad de autoaplicación a estudiantes de cursos que implementaron A+S entre los años 2015 y 2016. Los principales resultados dan cuenta de diferencias en la valoración que los estudiantes le asignan tanto al aprendizaje como al servicio. Esto respondería a las características de los proyectos, ya sea en su planificación, diseño o ejecución.

\section{Palabras clave}

Aprendizaje-servicio, educación superior, intervención social. 


\title{
Benefits of service learning experiences from the perspective of students at the University Alberto Hurtado
}

\begin{abstract}
Service-learning usually refers to a pedagogical approach that seeks to provide students with learning in a given subject, as well as to develop a service that meets the real needs of a community. This methodology is informed by pedagogical trends such as experiential learning and constructionism. However, the nature of servicelearning also suggests that it shares the characteristics of a social intervention. The present study addressed the research question: how do UAH students evaluate the benefits of service learning, after having had such an experience between 2015 and 2016? The aim of this paper is to describe the evaluation that UAH students make of the benefits of service-learning, both for their own training and for social organizations. This is a descriptive exploratory study with mixed methodology, using a semi-structured questionnaire, with self-application, to students of degree programmes that implemented service-learning between 2015 and 2016. The main results show differences in the evaluation that students give to projects, both in terms of learning and the service performed, depending on the characteristics of the projects, whether in their planning, design or execution.
\end{abstract}

\section{Keywords}

Service-learning, higher education, social intervention. 


\section{Introducción}

Hablar de aprendizaje-servicio es referirse a una propuesta educativa que, como señala la literatura científica, aporta y vincula dos componentes centrales: por un lado, hace posible que los aprendizajes se produzcan de modo contextualizado, que el alumnado desarrolle las capacidades de crítica, de reflexión, de participación ciudadana activa (Folgueiras, Luna y Puig, 2013) y por otro lado, se vincula e impacta en la comunidad con un servicio concreto. En la Universidad Alberto Hurtado (en adelante UAH), institución educativa chilena perteneciente a la Compañía de Jesús, esta estrategia comenzó a difundirse desde el año 2008. Luego de su primera fase piloto de implementación entre 2008 y 2011, desde el 2012 comenzó a expandirse en las seis facultades, desarrollándose más de 100 proyectos en total. Este aumento de cursos con aprendizaje servicio (en adelante $A+S$ ), implicó el desafío de evaluar los resultados de esta implementación. Esta trabajo nace de la reflexión por comprender el aporte de la metodología aprendizaje servicio desde la perspectiva de los estudiantes que participaron de esas iniciativas. Este estudió describirá y analizará los resultados de evaluación de estudiantes que cursaron asignaturas A+S entre los años 2015 y 2016.

La investigación en aprendizaje-servicio es incipiente en Chile, por tanto la relevancia de este trabajo radica en que ofrece, a nivel teórico-disciplinar, una oportunidad para analizar los resultados de la aplicación de $A+S$, específicamente en la Universidad Alberto Hurtado y desde ahí pensar la evaluación de las experiencias de aprendizaje-servicio como un proceso continuo de mejora para futuras implementaciones, tanto del aprendizaje (la formación de los estudiantes), como en el servicio. Esto se hará desde la óptica de la intervención social, concepto que tiene una tradición desde la psicología comunitaria (Alfaro, 2000; Sánchez, 2007) en tanto mecanismo que se centra en problemas, necesidades 0 cuestiones sociales detectados en un sistema social.

\section{Marco teórico}

\subsection{Educación Superior en Chile}

Desde la creación en 1842 de la Universidad de Chile, la historia de la Educación Superior chilena fue dominada por el Estado, incluso después de que universidades privadas comenzaron a emerger hacia fines del siglo XIX y en la primera mitad del siglo XX (Bernasconi, 2003). En el transcurso de la década de 1960, la universidad se transformó para algunos en un medio de influencia e intervención de la universidad en la sociedad. Así entonces, el rol de la extensión dentro de las universidades tendió a trascender los límites de la acción académica y comenzó a sumergirse en el campo de lo político-contingente. La extensión pasó a ser un instrumento que superó el quehacer propiamente universitario. Esto se agudizó con la Reforma Universitaria, un proceso de intensa discusión sobre la naturaleza de

Caire, M. (2019). Contribuciones de las experiencias de aprendizaje-servicio desde la perspectiva de estudiantes de la Universidad Alberto Hurtado. RIDAS, Revista Iberoamericana de Aprendizaje Servicio, 7, 89-111. DOI10.1344/RIDAS2019.7.6 
la universidad y su papel en la sociedad chilena. Posteriormente, en Dictadura,

"la política de extensión tuvo por objetivo principal trasmitir el saber que se generaba al interior de la universidad, fundamentalmente, a través del perfeccionamiento, capacitación y especialización para aquellos que aplican estos adelantos al desarrollo material y cultural de la nación" (Donoso, 1993, p. 185).

Con el retorno a la democracia, la Educación Superior en Chile transitó de una estructura centrada en desafíos productivos a otra en que el conocimiento es el motor del cambio y progreso (UNESCO, 1998) y en donde la educación contemporánea cumple un rol crucial en términos de formar profesionales competentes académicamente y comprometidos con el desarrollo social (Tapia, 2008; Batlle, 2013). En Chile, la Comisión Nacional de Acreditación (CNA) postula que la nueva forma en que las universidades se han de vincular con el medio debiera transitar desde un modo tradicional, caracterizado principalmente por una relación unidireccional desde la universidad hacia el medio, a un modo bidireccional. Ese modo bidireccional busca la interacción entre Universidad y Comunidad, considerando la simetría entre quienes son parte de esa interacción. Una forma posible de alcanzar esto es a través de $A+S$, una forma de vinculación con el medio que favorece la formación de universitarios que se forman con una serie de habilidades como el compromiso social, el trabajo en equipo, comprometidos con la resolución de problemas sociales y con un profundo sentido crítico en torno a la sociedad (Stanton, 1990).

\subsection{Aprendizaje Servicio en la Universidad Alberto Hurtado}

La Universidad Alberto Hurtado (UAH), institución de educación superior perteneciente a la Compañía de Jesús, busca en su proyecto formativo expresar su sello humanista marcado por una particular sensibilidad y preocupación por el ser humano. El sentido último de la formación en la UAH es contribuir al desarrollo integral de sus estudiantes, de modo que como futuros profesionales sean un aporte en la transformación de la sociedad (Universidad Alberto Hurtado, 1998). Es así que, la UAH en el año 2008 inicia el programa $A+S$, dirigido desde el Centro de Reflexión y Acción Social (CREAS), que tenía la misión de promover la Responsabilidad Social Universitaria según el enfoque de AUSJAL. La primera etapa de implementación ocurrió entre los años 2008-2011. En esos años se desarrollaron 28 cursos con la metodología $A+S$ y se trabajó con 16 socios comunitarios. Algunos de estos cursos se implementaron en una modalidad piloto, con la lógica de evaluar sus resultados en términos de obstáculos y/u oportunidades de desarrollo. Desde el año 2012 se inició una segunda fase de expansión del $A+S$ en la UAH. Con ello, varias unidades académicas iniciaron un proceso de revisión de sus mallas curriculares, que derivó en la elección de nuevas asignaturas que implementaron la metodología (ver Tabla 1).

Tabla 1: Número de cursos A+S UAH entre los años 2008 - 2016 


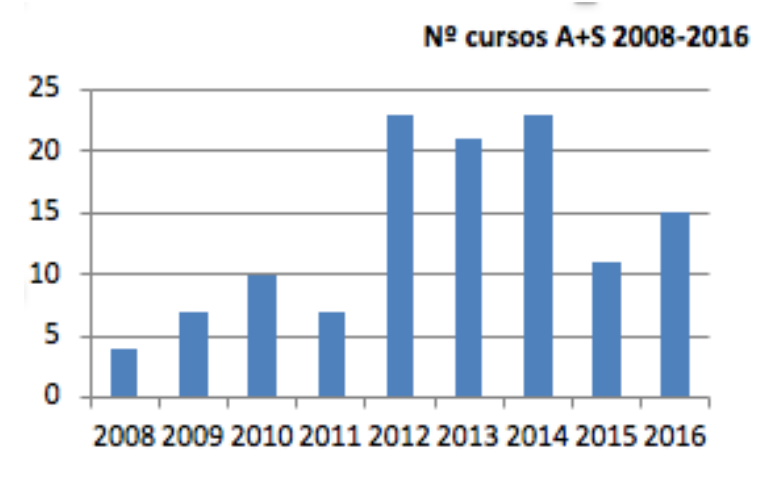

Fuente: Elaboración propia

A partir de la historia y experiencia acumulada en años de trabajo del Centro de Ética y del Centro de Reflexión y Acción Social (CREAS) nace el año 2015 el Centro de Ética y Reflexión Social Fernando Vives SJ, perteneciente a la Vicerrectoría de Integración de la $\mathrm{UAH}$, a partir de la fusión del CREAS y el Centro de Ética UAH. El Centro de Ética y Reflexión Social es un Centro de Investigación de la Universidad Alberto Hurtado. Su trabajo se desarrolla desde una perspectiva plural y respetuosa del diálogo.

Una de las líneas de trabajo del centro es implementar la metodología $A+S$ como propuesta coherente con el proyecto formativo y el modelo pedagógico de la UAH. La fusión de ambos centros implicó ese año una disminución de los cursos $A+S$ como una medida preventiva mientras se ajustaban las labores del equipo.

\subsection{Aprendizaje-servicio: definición y} algunos rasgos distintivos

$A+S$ es entendido como una forma de enseñanza-aprendizaje que involucra a estudiantes en el desarrollo de un servicio en la comunidad para alcanzar sus objetivos académicos (Furco y Billig 2002; Tapia, 2006; Batlle, 2013). Si bien el concepto de aprendizaje-servicio -service-learning- se utilizó explícitamente por primera vez en el South Regional Education Board (Giles y Eyler, 1994), esta definición es fruto de tradiciones anglosajonas y latinoamericanas que posiblemente inciden en la forma en que se desarrollan este tipo de prácticas de vinculación formal entre el curriculum y acciones solidarias.

Butin (2010) afirma que el $A+S$ se formó en el fragor de los movimientos por los derechos civiles en Estados Unidos en la década del 60. Esto, junto con otras leyes y reformas educativas, impulsaron explícitamente el Voluntariado Universitario y el aprendizaje-servicio curricular. Desde esta tradición, las investigaciones reportan que el $A+S$ contribuye en los estudiantes a la comprensión de los problemas sociales y su capacidad para aplicar lo visto en clases, siendo el compromiso cívico uno de los principales resultados (Ann et al., 2014).

Desde la tradición latinoamericana del aprendizaje-servicio, habría que mencionar la creación del Servicio Social en las universidades de México a principios del siglo XX (Tapia, 2006; Ochoa, 2010). En paralelo, un gran número de iniciativas solidarias desarrolladas desde las escuelas y las instituciones de educación superior, en los años 70, comenzaron a multiplicarse tanto voluntarias como obligatorias (Ochoa, 2010). Paralelamente, creció en la región el interés por la 
metodología del aprendizaje-servicio. En Argentina desde 1996 se desarrolla a nivel nacional una política de promoción explícita del aprendizajeservicio en el sistema educativo (Ochoa, 2010).

En Chile, el año 2000 comenzó a promoverse el $A+S$ desde las políticas educativas nacionales, a través de la Red Construye País. En este contexto, comienzan las primeras experiencias que explícitamente se denominaron $\mathrm{A}+\mathrm{S}$. Si bien algunos docentes de diferentes universidades comenzaron con implementaciones de aprendizajeservicio en sus cátedras, estos esfuerzos fueron aislados y sin un compromiso institucional detrás. Fue la Pontificia Universidad Católica de Chile la que comenzó en el año 2004 con un programa formal de apoyo a la implementación de estas experiencias en su institución (Berríos, Contreras, Herrada, Robles y Rubio, 2012), recogiendo la trayectoria norteamericana para generar su modelo $A+S$. Luego el Programa Liceo para Todos y posteriormente el Premio Bicentenario Escuelas Solidarias entre los años 2006 y 2009, fueron las iniciativas pioneras de $A+S$ en Chile. En el año 2011 se constituyó la Red Nacional de Aprendizaje-Servicio (REASE), que busca promover esta estrategia tanto en el nivel escolar como en educación superior.

$\mathrm{El} A+\mathrm{S}$ tiene sus bases teóricas en el ámbito educacional. Dos autores que han influido en la configuración de este enfoque son John Dewey y Paulo Freire (Tapia, 2008). El aprendizaje experiencial brinda una perspectiva holística e integradora del aprendizaje que combina experiencia, percepción, cognición y comportamiento (Kolb, 1993). Las definiciones de Dewey sobre la educación, enfatizan los factores culturales tales como la participación cívica, la comunicación y la vida comunitaria (Deans, 1999).

Desde los aportes de Paulo Freire, particularmente desde la Pedagogía de la Liberación, la educación se encuentra en la encrucijada de ser parte de los procesos emancipatorios de los más excluidos y marginados de la sociedad. En ese sentido:

"Tanto Dewey como Freire son humanistas que ven el proceso educativo en tanto acción y reflexión, aunque con un cierto matiz. Entre lo más significativo es que Freire hace hincapié en un enfoque antropológico hacia los estudiantes, representando su cultura, clase y raza - factores que Dewey elude en gran medida cuando habla de los estudiantes" (Deans, 1999, p. 20).

Otros aportes que han ayudado a construir este enfoque de aprendizajeservicio lo ofrece el construccionismo. Desde los planteamientos de Berger y Luckmann (1967), la realidad se construye socialmente, la comprensión del mundo depende de una miríada de influencias: sociales, culturales, económicas, políticas e históricas.

Mucha evidencia ha demostrado los impactos que tiene $A+S$ en la formación académica de los estudiantes. En términos de aprendizajes, se evidencia el aumento en las calificaciones de los estudiantes; la aplicación concreta de los contenidos curriculares en ámbitos 
reales; pensamiento de orden superior, análisis de problemas y pensamiento crítico. En el ámbito profesional el aprendizaje servicio contribuye al desarrollo de las carreras; mayor motivación por la disciplina que estudian; y la relación con la institución; son más propensos a graduarse; aumenta la tasa de retención y adquieren mayor cercanía con la institucionalidad (Bringle y Hatcher, 1996; Eyler y Giles, 2001; Furco y Root, 2010).

En Chile existen pocos estudios relacionado con los resultados de experiencias de $A+S$. Para efectos de indagar sobre los resultados del aprendizaje-servicio en la Universidad Alberto Hurtado, es necesario describir la evaluación que hacen los estudiantes acerca del aporte del aprendizajeservicio.

\section{Metodología}

\subsection{Diseño de estudio}

Se trató de un estudio exploratorio descriptivo (Hernández, Fernández y Baptista, 2003) que se realizó con metodología mixta cuantitativa y cualitativa, utilizando un cuestionario con preguntas abiertas y cerradas con modalidad de autoaplicación masiva a los estudiantes de un grupo de cursos que implementaron aprendizaje-servicio entre los años 2015 y 2016.

La fase cuantitativa del presente trabajo implicó la realización de análisis descriptivos sobre los porcentajes de acuerdo expresados por los participantes de diferentes proyectos $A+S$, frente a diversas afirmaciones referidas al grado de contribución de $A+S$ a su formación personal y a la comunidad. Para ello se construyeron múltiples tablas de contingencia bivariadas, con las cuales se buscó determinar la medida en que los porcentajes de acuerdo expresados por los encuestados variaron dependiendo del proyecto analizado. Esto permitió identificar los proyectos más valorados en términos de los porcentajes de acuerdo alcanzados.

La fase cualitativa implicó la revisión de las dos preguntas abiertas del cuestionario: ¿Cuál fue el o los aprendizajes más importantes que desarrollaste en esta actividad? ¿Qué sugerencias harías para próximos cursos con esta modalidad?. Basando el análisis en la Teoría Fundada (Strauss y Corbin, 2002) se identificaron algunas categorías emergentes en base a una codificación abierta.

\subsection{Participantes}

El universo estaba conformado por estudiantes de ambos sexos y de distintas carreras de la UAH, que participaron de cursos con aprendizajeservicio entre los años 2015 y 2016. La muestra de los cursos ha sido intencional, tomando en consideración las aportaciones de Tapia (2006) para la selección de proyectos de aprendizajeservicio, según criterios que gozan de mayor consenso a nivel internacional, para significar un proyecto consolidado de aprendizaje-servicio. Los estudiantes seleccionados debían haber participado en proyectos que cumplieran con los siguientes requisitos:

1. Proyectos que estuvieran integrados

Caire, M. (2019). Contribuciones de las experiencias de aprendizaje-servicio desde la perspectiva de estudiantes de la Universidad Alberto Hurtado. RIDAS, Revista Iberoamericana de Aprendizaje Servicio, 7, 89-111. DOI10.1344/RIDAS2019.7.6 
en el currículum.

2. Proyectos que ofrecieran un servicio a la comunidad.

3. Proyectos que estuvieran articulados con organizaciones, entidades sociales, de la sociedad civil, denominados socios comunitarios en el contexto nacional chileno.

A continuación, una breve descripción de los cursos seleccionados:

Proyecto 1. Consiste en la participación de estudiantes de $2^{\circ}$ año de una carrera del área de Ciencias Sociales, los cuales realizan un servicio de investigaciones a socios comunitarios que tratan con grupos de riesgo de exclusión social.

Proyecto 2. Consiste en la participación de estudiantes de $3^{\circ}$ año de una carrera del área de Ciencias Sociales, los cuales realizan un servicio de levantamiento de información de una zona semi rural de la Región

Metropolitana, trabajando directamente en vinculación con actores de un municipio, junta de vecinos y una fundación.

Proyecto 3. Consiste en la participación de estudiantes de $3^{\circ}$ y $4^{\circ}$ año de una carrera del área disciplinar de Ciencias Sociales, los cuales realizan un servicio de asesoría en el diagnóstico y diseño de un plan de intervención, a funcionarios municipales, de gobierno o entidades sociales que trabajen aplicando políticas públicas.

Proyecto 4. Consiste en la participación de estudiantes de $3^{\circ}$ año de una carrera del área de Humanidades, los cuales realizan un servicio a microempresas.

Proyecto 5. Consiste en la participación de estudiantes de $4^{\circ}$ año de una carrera de Ciencias Sociales, los cuales realizan un servicio de evaluación de programas sociales.

Proyecto 6. Consiste en la participación de estudiantes de un curso optativo del área de Humanidades, los cuales realizaron un servicio de nivelación de español para migrantes.

En consecuencia, la muestra la integran los estudiantes participantes de seis cursos con $A+S$, pertenecientes a dos área disciplinares, durante los años 2015 y 2016 . En la Tabla 2 se explicita el número de estudiantes que respondieron la encuesta.

Tabla 2: Número de estudiantes que respondieron encuesta según curso de la muestra

\begin{tabular}{|l|l|l|l|}
\hline $\begin{array}{l}\text { Área disciplinar de los } \\
\text { estudiantes }\end{array}$ & $\begin{array}{l}\text { Año de } \\
\text { imple- } \\
\text { men- } \\
\text { tación }\end{array}$ & $\begin{array}{l}\text { No } \\
\text { estu- } \\
\text { dian- } \\
\text { tes del } \\
\text { curso }\end{array}$ & $\begin{array}{l}\text { No } \\
\text { estu- } \\
\text { dian- } \\
\text { tes } \\
\text { que } \\
\text { res- } \\
\text { pon- } \\
\text { den }\end{array}$ \\
\hline $\begin{array}{l}\text { Ciencias Sociales } \\
\text { (proyecto 1) }\end{array}$ & 2015 & 60 & 51 \\
\hline $\begin{array}{l}\text { Ciencias Sociales } \\
\text { (proyecto 2) }\end{array}$ & 2015 & 29 & 16 \\
\hline $\begin{array}{l}\text { Ciencias Sociales } \\
\text { (proyecto 3) }\end{array}$ & 2015 & 40 & 22 \\
\hline $\begin{array}{l}\text { Humanidades } \\
\text { (proyecto 4) }\end{array}$ & 2016 & 25 & 25 \\
\hline
\end{tabular}




\begin{tabular}{|l|l|l|l|}
\hline $\begin{array}{l}\text { Ciencias Sociales } \\
\text { (proyecto 5) }\end{array}$ & 2016 & 24 & 20 \\
\hline $\begin{array}{l}\text { Humanidades } \\
\text { (proyecto 6) }\end{array}$ & 2016 & 18 & 8 \\
\hline Total & & 196 & 141 \\
\hline
\end{tabular}

Fuente: elaboración propia

\subsection{Instrumento y variables}

Este instrumento fue creado el año 2009 como una adaptación de un instrumento de evaluación aplicado en cursos $A+S$ de la Pontificia Universidad Católica de Chile. Durante el año 2014, se revisó dicho instrumento de evaluación, que constaba de 22 preguntas con alternativas tipo likert y 2 preguntas abiertas. Tomando en consideración est instrumento, se socializó con representantes de las 6 facultades de la UAH la pertinencia de la encuesta, obteniendo retroalimentación para la mejora (Caire, 2015). A esto se sumó la revisión de un representante de la Dirección de Docencia de la UAH, para estar alineados con lo que la universidad recoge en sus propias evaluaciones docentes. De esta manera, el cuestionario para estudiantes quedó conformado por 14 preguntas con alternativas tipo likert y 2 preguntas abiertas.

Se realizó un análisis del instrumento de evaluación $A+S$, que fue sometido a un test de confiabilidad según las 4 dimensiones incluidas en la medición. El análisis de confiabilidad a través de alfa de Cronbach indica que las dimensiones generadas tienen una alta consistencia interna $(a>0,6)$ para los cuatro indicadores, como se observa en la Tabla 3.

Tabla 3: Coeficientes alfa de Cronbach para indicadores generales

\begin{tabular}{|l|l|l|l|l|}
\hline & $\begin{array}{l}\text { Forma- } \\
\text { ción } \\
\text { Perso- } \\
\text { nal }\end{array}$ & $\begin{array}{l}\text { Vincu- } \\
\text { lación } \\
\text { con el } \\
\text { medio }\end{array}$ & $\begin{array}{l}\text { Rela- } \\
\text { ción } \\
\text { con el } \\
\text { Socio } \\
\text { Comu- } \\
\text { nitario }\end{array}$ & $\begin{array}{l}\text { Rela- } \\
\text { ción } \\
\text { con el } \\
\text { Do- } \\
\text { cente }\end{array}$ \\
\hline $\begin{array}{l}\text { Alfa de } \\
\text { cronbach }\end{array}$ & 0,845 & 0,636 & 0,855 & 0,862 \\
\hline $\begin{array}{l}N^{\circ} \text { de } \\
\text { ítems }\end{array}$ & 4 & 3 & 4 & 3 \\
\hline
\end{tabular}

Fuente: elaboración propia

\section{Resultados}

\subsection{Resultados del análisis cuantitativo}

En relación a la dimensión Formación Personal, se evidencia que los encuestados tienen, en general, una alta valoración de la experiencia $A+S$. El proyecto 4 tienen en todos los ítems una valoración positiva superior al promedio de todos los proyectos. Le sigue el proyecto 3 que tiene en 3 items una valoración positiva superior a la media. Por el contrario, el proyecto 5 tiene en los 4 items una valoración bajo el promedio de todos los proyectos y el proyecto 2 también, aun cuando los valores son altos.

Considerando los 3 items relacionados con la dimensión Vinculación con el medio social, es posible evidenciar que los estudiantes tienen, en general, una valoración promedio menor respecto de la dimensión de Formación Personal. El proyecto 6 tiene en todos los ítems una 
valoración positiva superior a la media de todos los proyectos. Le sigue el proyecto 4 que tiene en 2 items una valoración positiva superior a la media. Por el contrario, el proyecto 1 tiene en los 3 items una valoración bajo la media respecto de todos los proyectos.

Respecto a la dimensión Relación con el Socio Comunitario, es posible evidenciar que los estudiantes tienen, en general, una valoración promedio menor respecto de las demás dimensiones del cuestionario. Esto es sin duda un hallazgo interesante, puesto que esta dimensión entrega información relevante para el análisis desde el punto de vista de la intervención social. Sobre sus resultados, los proyectos 3 y 6 tienen en todos los ítems una valoración positiva superior a la media respecto de todos los proyectos. Finalmente, en la dimensión Rol del equipo docente es posible evidenciar que los estudiantes tienen, en general, una alta valoración de esta dimensión. El proyecto 1 tiene en 2 de los ítems una valoración positiva superior a la media respecto de todos los proyectos.

A partir de los resultados, se presenta una síntesis por dimensión, ítems y proyectos. Respecto a las 4 dimensiones, es posible ver en la Tabla 4 que hay proyectos ( 1 y 2 ) que tienen una alta valoración en los aspectos relacionados con el aprendizaje, no así con los aspectos relacionados al servicio, así como hay también hay proyectos ( 3 y 6 ) que tienen una alta valoración en ambos aspectos.

Tabla 4: Porcentaje de acuerdo (considerando respuestas totalmente de acuerdo + de acuerdo) según dimensiones por Proyecto.

\begin{tabular}{|l|l|l|l|l|}
\hline & $\begin{array}{l}\text { Formación } \\
\text { Personal }\end{array}$ & $\begin{array}{l}\text { Vinculación } \\
\text { con el } \\
\text { medio } \\
\text { social }\end{array}$ & $\begin{array}{l}\text { Relación } \\
\text { con } \\
\text { Socio } \\
\text { Comuni- } \\
\text { tario }\end{array}$ & $\begin{array}{l}\text { Rol } \\
\text { equipo } \\
\text { docente }\end{array}$ \\
\hline 1 & $80,7 \%$ & $39,4 \%$ & $59,1 \%$ & $92,4 \%$ \\
\hline 2 & $82,4 \%$ & $64,5 \%$ & $42,1 \%$ & $90,0 \%$ \\
\hline 3 & $87,5 \%$ & $62,5 \%$ & $89,1 \%$ & $79,2 \%$ \\
\hline 4 & $98,0 \%$ & $76,0 \%$ & $79,0 \%$ & $97,3 \%$ \\
\hline 5 & $71,3 \%$ & $67,5 \%$ & $57,5 \%$ & $85,0 \%$ \\
\hline 6 & $84,4 \%$ & $79,2 \%$ & $81,3 \%$ & $79,2 \%$ \\
\hline
\end{tabular}

Fuente: elaboración propia

Finalmente como síntesis de los resultados cuantitativos, es interesante revisar lo que sucede a nivel de proyectos. Comparando los 6 proyectos en los 14 ítems del cuestionario, el Proyecto 4 tiene una valoración positiva superior al promedio de cada pregunta en 10 de los 14 items. Esto quiere decir que en casi todas las preguntas, este proyecto tuvo una valoración positiva más alta, comparada con los otros proyectos, siendo la Dimensión Formación Personal en la que se encuentra mejor evaluada. Por el contrario, el proyecto 5 tiene una valoración positiva inferior al promedio de cada pregunta en 6 de los 14 items. Esto quiere decir que en casi la mitad de las preguntas este proyecto tuvo una valoración positiva más baja comparada con los otros proyectos, siendo la Dimensión Formación Personal en la que se encuentra con la menor evaluación, comparada con el resto de 
los proyectos.

\subsection{Resultados del análisis cualitativo}

De la muestra total de 142 estudiantes, 120 respondieron estas preguntas. Los resultados del apartado cualitativo se muestran en orden a las dos preguntas: i) los aspectos más significativos de esta experiencia $A+S$, ii) sugerencias para próximos cursos $A+S$.

i) Aspectos más significativos de la experiencia $A+S$

De acuerdo a la revisión de las respuestas abiertas del cuestionario, las grandes categorías que destacan los estudiantes son el conocimiento del ámbito laboral como resultado de $\mathrm{A}+\mathrm{S}$; la relación con la comunidad; salir de la sala de clases como encuentro con la realidad; relación teoría/práctica en el $A+S$ y el desarrollo de habilidades.

En primer lugar, el ámbito laboral como resultado del aprendizaje-servicio.

Desde el punto de vista de los participantes, el aprendizaje-servicio tiene una alta valoración, en tanto posibilidad de conectarse con el mundo laboral, con lo que ellos suponen, podría ser un espacio de futuro trabajo en el cual desempeñarse. Dicho de otro modo, la posibilidad de la experiencia $A+S$ como una primera vez frente a su futuro campo laboral, siendo ésta una de las dimensiones con mayor aceptación entre los estudiantes. Esto implica visualizarse profesionalmente en esos espacios, es decir, visualizar el futuro laboral en tanto comprensión del sentido de la carrera que estudian.

\section{"...Por otro lado, generar una ayuda}

consolidada a la organización sirve como experiencia a la hora de entrar al mundo laboral, ya que nos acerca a una realidad distinta, a nivel organizacional" (estudiante mujer, 23 años).

"Enfrentarse a una experiencia concreta y una posible proyección laboral" (estudiante hombre, 23 años).

En segundol lugar, la relación con la comunidad. Un rasgo distintivo en $\mathrm{A}+\mathrm{S}$ es desarrollar un servicio destinado a atender necesidades de una comunidad. Desde el punto de vista de los estudiantes, la comunidad, entendida en la metodología como el socio comunitario, la contraparte de la entidad educativa, juega un rol clave a la hora de comprender el valor que tiene para los estudiantes, participar de estas experiencias. En ese sentido, las respuestas en esta dimensión tienden a valorar la interacción que se da con esa contraparte, el acercamiento con personas que son externas a su realidad cotidiana en el espacio universitario:

"La vinculación con los grupos sociales en el terreno, permitió adentrarme de mejor forma con las realidades de esta comunidad" (estudiante hombre, 22 años).

Hay otros estudiantes que entienden esta relación no solo como un estar con las personas, sino como un vincularse e implicarse con ellas. Esto habla de un entendimiento más profundo sobre las necesidades de la gente, en tanto personas con quienes establecer una forma de 
trabajo:

"El trabajo práctico y en terreno en conjunto con una contraparte, puesto que nos ayudó a hacernos una idea del trabajo concreto a realizar como profesionales en la posteridad" (estudiante hombre, 22 años).

En tercer lugar, salir de la sala de clase: el encuentro con la realidad. Un aspecto señalado en las respuestas abiertas de los estudiantes es el valor que le asignan a salir de los márgenes de la universidad y encontrarse con otros espacios, muchos de ellos en contacto con una realidad diferente a la de ellos o a la que frecuentan habitualmente. Comprenden que el valor de salir de la sala de clases está en contactarse y comprender la realidad, comprender la complejidad de lo social y los cambios que se requieren a nivel social e incluso a nivel de personas. Salir de la sala de clases implica mayor conciencia de los problemas desde una visión más crítica:

"Poder ver realidades sociales externas a mi realidad y poder ver los problemas existentes" (estudiante mujer, 19 años).

"Dejar de ser un estudiante y pasar a la puesta en práctica de los aprendizajes, sí mismo analizar y comprender la complejidad social actual chilena y los cambios que se necesitan realizar en esta" (estudiante hombre, 22 años).

En cuarto lugar, la relación teoría/práctica en el $A+S$. Quizás uno de los aspectos más distintivos del aprendizaje-servicio es llevar los conocimientos a la práctica.

Discursivamente se presenta así en gran parte de las definiciones de la metodología. Esto es parte de lo que aparece en los discursos de los estudiantes, al señalar que uno de los aspectos más significativos fue esto que permitió poner en práctica los conocimientos:

"Comprobar que la materia vista en clases si se puede aplicar en la vida real. Porque muchas veces pensamos que nunca vamos a aplicar lo que aprendemos en clases y este curso fue la prueba de que no es asi" (estudiante hombre, 20 años).

Esta relación teoría/práctica, para algunos estudiantes, no es solo aplicar conocimientos, también la entienden como una contribución a una comunidad:

"El poder aplicar conocimientos, hace que nos formemos como profesionales y podamos contribuir a crear comunidad" (estudiante mujer, 20 años).

Finalmente, el desarrollo de habilidades como fruto del trabajo. No es nuevo decir que el aprendizaje-servicio significa para los estudiantes una serie de habilidades que se desarrollan. Al respecto, los estudiantes participantes de estos proyectos, destacan la responsabilidad y compromiso como habilidades significativas en estas experiencias:

"La responsabilidad que implica realizar un proyecto que no solo me involucre como persona a mi sino que tenga que responder ante otras 
instituciones" (estudiante hombre, 19 años).

"Los aspectos, según mi experiencia, fue el hecho de llevar a la práctica todo lo que aprendimos en el semestre pero no tan solo la materia sino también poder de nuestra responsabilidad y compromiso" (estudiante mujer, 21 años).

Algunos estudiantes reportaron que el trabajo en equipo fue una de las habilidades más destacadas, esto puede deberse al tipo de relación que se establece a lo largo del proyecto:

"El trabajo en equipo y la experiencia práctica que esto significa, ya que cuando se trabaja en campo las condiciones de todo cambian" (estudiante mujer, 19 años).

ii) Sugerencias para los próximos cursos $\mathrm{A}+\mathrm{S}$

De acuerdo a la revisión de las respuestas del cuestionario, las grandes categorías que destacan los estudiantes son: equilibrio entre necesidades sociales y aprendizajes; comunicación con la comunidad; más tiempo en terreno; consideraciones de carga en el quehacer de $\mathrm{A}+\mathrm{S}$.

En primer lugar, la comunicación con la comunidad. Un aspecto que apareció con fuerza en el reporte de los estudiantes como aspectos a mejorar es la comunicación que se estableció con la comunidad, puesto que, en algunos casos, pareciera ser que la participación de las contrapartes no fue activa para el logro de los acuerdos, que la información que entrega el socio comunitario sea más clara o bien, que la comunicación sea más fluida la comunicación y por último, que el socio comunitario esté bien constituido para facilitar el trabajo:

"Sería oportuno garantizar la existencia de una contraparte bien constituida, la cual sea verdaderamente un aporte desde el comienzo para la vinculación con la realidad a intervenir. Esto pues, de no ser así, como lo fue en mi experiencia, dificulta el desarrollo del trabajo" (estudiante hombre, 24 años).

En segundo lugar: más tiempo en terreno. Desde el punto de vista de los estudiantes, hay una tensión respecto al factor tiempo. Algunos participantes destacan que se requiere más tiempos para desarrollar un servicio de calidad, más terrenos o incluso señalan, que el curso tenga una mayor duración. Otros estudiantes en cambio señalan que el trabajo sea más ajustado al tiempo del semestre académico, para realizarlo de buena manera:

"Creo que el tiempo tan reducido que tiene el semestre se transforma en un obstáculo a la hora de realizar un trabajo tan amplio y que abarca tantos contenidos. Tal vez si el trabajo fuera un poco más acotado se lograría hacer algo con mayor calidad en un periodo corto de tiempo" (estudiante mujer, 22 años).

En tercer lugar, consideraciones de carga en el quehacer de $A+S$. Un aspecto que algunos estudiantes consideraron necesario replantear, es la carga que significó el trabajo, ya sea en 
términos académicos

(fundamentalmente temporales y de evaluaciones) así como algunos casos señalados en términos económicos:

"Creo que tiende a ser un trabajo muy arduo. Es algo que no me esperaba tomase tanto tiempo" (estudiante mujer, 20 años).

"Que tomen en cuenta los gastos monetarios y la carga académica en la que se encuentran los futuros estudiantes" (estudiante mujer, 19 años).

\section{Discusión}

5.1 Aportes del aprendizaje-servicio a la formación de los estudiantes

Lo primero a destacar es la alta valoración que tienen los estudiantes respecto del aporte que entrega $A+S$ en su formación. De acuerdo a los datos, la dimensión formación personal tiene un promedio sobre el $83 \%$ de valoración positiva.

Distintos elementos del $A+S$ podrían estar impactando en la formación de los estudiantes. De acuerdo a los resultados que se presentaron anteriormente, los estudiantes destacan la conexión de la experiencia con un ámbito de acción profesional. Estas experiencias cumplen con los propósitos programáticos de desarrollar en los estudiantes habilidades profesionales y académicas, que contribuyen a su formación integral, siendo percibidas como útiles para el futuro laboral. Tal como indican Furco y Billig (2002), entre los efectos positivos de participar en proyectos $A+S$, se encuentran la ampliación de la conciencia y de las opciones vocacionales, la mejora de las competencias profesionales, la mayor comprensión con la ética del trabajo y una mayor preparación para el mundo del trabajo. En ese sentido, el $A+S$ resulta una buena aproximación laboral que los acerca a un área de desarrollo profesional concreto.

Esto es relevante, considerando que en las instituciones de educación superior, uno de los grandes desafíos es la inserción laboral de sus egresados, muchos de ellos con poca experiencia previa en espacios laborales reales, tal como indican Fernández, Ceinos y Nogueira (2017). La preparación para el ejercicio profesional, la toma de decisiones o la promoción de la empleabilidad son aspectos del desarrollo profesional $y$, aunque dependerá de las decisiones que tome cada persona y de cómo construya su futuro profesional, las instituciones de educación superior no pueden quedan al margen de las responsabilidades institucionales a la hora de contribuir a crear oportunidades efectivas de desarrollo profesional.

Otro elemento destacado por los estudiantes es la valoración que hacen de $A+S$ respecto a su contribución en el desarrollo de habilidades profesionales como la responsabilidad social, el compromiso y el trabajo en equipo.

Esto es consistente con las finalidades del Proyecto Formativo UAH (2008) que aspira a formar a sus estudiantes para la excelencia académica y profesional, para la justicia social, el servicio, que sean reflexivos, críticos y que asuman funciones con un profundo sentido ético y de responsabilidad. En la misma 
línea, los planteamientos de Eyler y Giles (1999) y Jouannet et al. (2013) indican que el $A+S$ es un importante precursor del desarrollo de habilidades transversales de los estudiantes y de su éxito académico, como el compromiso social, la resolución de problemas, el trabajo en equipo y las habilidades comunicativas.

Algo común en los seis proyectos es que en todos valoran la posibilidad de relacionar los conocimientos aprendidos en el curso con una realidad. Esto es coherente con otras investigaciones, que destacan que uno de los aspectos más valorados por los estudiantes es la posibilidad de pasar de la teoría a la práctica (Aramburuzabala y García, 2013; Folgueiras, Luna y Puig, 2013). Esto podría tener un impacto directo no solo en el desarrollo personal, sino académico y vocacional (Furco y Billig, 2002; Furco, 2003; Jouannet, Salas y Contreras, 2013; Opazo, 2015). Esta valoración del contacto con una realidad podría afectar el desarrollo de la responsabilidad social, en tanto se relaciona con la comprensión de problemáticas sociales y de la toma de conciencia del impacto social que pueden generar como futuros profesionales.

Ahora bien, cuando las experiencias son valoradas para el aprendizaje ¿son también percibidas como útiles para el socio comunitario? Desde la perspectiva de los estudiantes, una experiencia de $A+S$ no necesariamente contribuye de la misma forma, o con el mismo impacto, al aprendizaje y al servicio. Respecto a los proyectos del estudio, hay casos que son altamente valorados tanto desde el aprendizaje como el servicio, pero hay proyectos que teniendo una alta valoración de los estudiantes respecto a su contribución para el aprendizaje, no tienen la misma valoración respecto al aporte del servicio. $Y$ en otro, no parece ser un aporte al aprendizaje, pero sí es muy importante en cuanto al aporte del servicio para el socio comunitario. En relación a estas diferencias, los datos hacen pensar que características particulares de las experiencias, de su planificación y de su implementación, influyen en la valoración de los estudiantes hacia aspectos de la formación y el servicio. Desde los resultados, por ejemplo, es posible pensar que dentro de las experiencias de $A+S$, hay casos en que la articulación entre objetivos de aprendizaje del curso y el servicio, está mejor lograda que en otros, pudiendo existir experiencias $A+S$ que se desarrollan de manera menos integrada con los objetivos del curso y por tanto su contribución al aprendizaje de los contenidos del curso no es tan significativa, y sin embargo, el servicio se enfoca en responder a las necesidades de la comunidad. Eso es algo que hace interesante al $A+S$ y a la vez sumamente desafiante, el tener que responder a distintos propósitos, entre ellos el aprendizaje curricular, la intervención social, la formación ética, la responsabilidad social y la vinculación con el medio.

\subsection{Contribuciones desde la} intervención social para el servicio

De acuerdo a los datos, es preciso destacar que la dimensión Relación con el Socio Comunitario, tiene una valoración menor respecto de las 
demás dimensiones del cuestionario, lo que sugiere que el servicio fue en promedio la arista con menor evaluación. Este hallazgo es interesante, porque invita a identificar aspectos que pueden ayudar a mejorar la contribución del servicio dentro de la metodología.

A propósito de los datos, parece ser que las características de los proyectos que podrían afectar la valoración que hacen los estudiantes respecto a la contribución del servicio en el $A+S$ se pueden identificar algunos rasgos de los proyectos estudiados que coinciden con los planteamientos de Tryon y Stoecker (2008) sobre criterios de calidad en la implementación de estos cursos: levantamiento de necesidades, ajuste de expectativas entre el curso $A+S$ y los objetivos de la organización, establecimiento de compromisos de trabajo entre los socios comunitarios y docentes con estudiantes, la efectividad de la comunicación y un número importante de encuentros entre estudiantes y socio comunitarios. Varios de estos rasgos se identificaron en los proyectos 3 y 6 , los cuales tuvieron en promedio, la más alta valoración en relación al servicio.

Por ejemplo, en relación al levantamiento de necesidades, el proyecto 6 se vinculó con una comunidad que tenía dificultades para insertarse en el ámbito laboral. Esto fue diagnosticado junto a una entidad del sector, quienes fueron el puente para detectar algunas problemáticas con anticipación al inicio del semestre y traducirlo a una oferta académica que fuera resuelta desde alguna actividad universitaria. Esto, analizado desde el instrumento de evaluación, tuvo una alta valoración de los estudiantes respecto a si el servicio fue un aporte al socio comunitario y si el socio comunitario requería del servicio (87,5\% de valoración en ambas preguntas). Esto sugiere pensar que a medida que las necesidades son detectadas con anticipación y ajustadas a las expectativas del curso, es más probable que el servicio sea adecuado y pertinente para la comunidad.

Sin embargo, con la misma intención de desarrollar servicios que respondieran necesidades reales y orientadas a colaborar en la solución de problemáticas comunitarias, otros proyectos tuvieron resultados más bajos respecto a la valoración del servicio. Este puede ser uno de los aspectos más interesantes de observar según lo señalado por los estudiantes en este estudio: la forma de establecer el vínculo con la comunidad (desde mayor comunicación y más visitas), podría condicionar el éxito de los proyectos $\mathrm{A}+\mathrm{S}$ y más concretamente, podría afectar la contribución que los proyectos $A+S$ se proponen hacia los socios comunitarios. Tal como señalan Schmidt y Robby (2002) sobre el valor del aprendizaje-servicio para la comunidad, o los estudios de Mabry (1998) y Dahan (2016) sobre el contacto con los beneficiarios del servicio por más de 15 horas, el presente estudio también muestra que el contacto entre estudiantes y comunidad es un factor determinante para la calidad del servicio. Es en este punto que el enfoque de intervención social puede ser un aporte al modelo de implementación de $A+S$ en la $U A H$, 
pues podría ofrecer nuevas formas de vinculación con la comunidad que aseguren, por un lado, impacto en el servicio desarrollado, y al mismo tiempo, impacto en la formación de los estudiantes. En muchos cursos de $A+S$ es posible compatibilizar las necesidades de cambio de los socios comunitarios con los objetivos de aprendizaje del curso. Desde el punto de vista de la intervención social, esto es posible en la medida que ambos se dirigen a objetivos comunes y cuando los objetivos de la intervención generan oportunidades de participación de la comunidad para conseguir los cambios (Krause y Jaramillo, 1998).

Evidentemente esta clave es uno de los elemento deseados en el $\mathrm{A}+\mathrm{S}$. Acá hay una contribución clara y explícita de la intervención, y más específicamente de la intervención comunitaria, que es la colaboración y participación de los agentes de la comunidad. El enfoque comunitario que puede tener una intervención constituye una herramienta para el diseño, implementación, seguimiento y evaluación de políticas, programas y proyectos de intervención social (Martínez, 2007).

El planteamiento de la intervención comunitaria incluye una verdadera interacción entre interventor (estudiantes) y miembros del grupo de la comunidad (socio comunitario), de forma que cada uno de ellos pueda cambiar su punto de partida y praxis comportamental (Sánchez, 1991). Aquí la intervención comunitaria ofrece un punto que $A+S$ recoge en sus cimientos conceptuales, pero que al parecer en su abordaje práctico no ha sido posible desarrollar. La utilización de mecanismos participativos que maximicen el protagonismo de la comunidad tendientes al desarrollo de recursos en las organizaciones comunitarias, dando valor a sus propias acciones para modificar sus condiciones de exclusión, hasta los límites compatibles con la eficiencia técnica de la intervención (Sánchez, 1991; Lapalma, 2001).

Este aspecto es sin duda un elemento a considerar para el avance de un $\mathrm{A}+\mathrm{S}$ más integrado con perspectivas comunitarias, que apunten al reconocimiento identitario, pues pone el acento en las demandas y particularidades de los territorios, los grupos y sus personas, no como antídoto único, sino como una respuesta acorde a sus propias necesidades. $Y$ es en la planificación participativa y estratégica del diseño de alternativas de acción que faciliten la viabilidad de intervenciones que potencien derechos y deberes de la población (Lapalma, 2001).

Ahora bien, como dice Martínez (2007), aplicar el enfoque comunitario a las intervenciones sociales no significa, necesariamente, trabajar en forma directa con la comunidad y sus organizaciones, como se piensa normalmente. Idea que también está en coherencia con los planteamientos de Krause y Jaramillo (1998) en que recuerdan que la intervención comunitaria puede darse a diferentes niveles de la organización social y en diferentes modalidades, dependiendo de la postura teórica, valórica y epistemológica que adoptan los actores del proceso. En ese sentido, esta 
participación dependerá también, de las características del proyecto, las demandas de la comunidad así como las características que la asignatura tenga. Fruto de ese análisis, será pertinente pensar hasta qué punto, la comunidad puede tener un rol clave a la hora de incidir en los proyectos $A+S$.

Tal como se señaló en el marco teórico, una intervención tiene un proceso interventivo que contempla algunas acciones clave: definir un objeto de intervención, identificar el estado inicial de la intervención, tipo de cambio perseguido, metas, ámbitos de aplicación, técnicas usadas y duración temporal (Sánchez, 1991). Con esto, cabe preguntarse en qué medida se nutre el modelo actual de $A+S$ desde la perspectiva de la intervención social, de tal manera de generar nuevas formas de entender (conocimientos) y actuar (técnicas) dirá Saavedra (2005).

Al revisar el panorama de proyectos $A+S$, de acuerdo con Sánchez (1986), se puede señalar que respecto al objeto o destinatario de la intervención, hay coincidencia en los 6 proyectos en trabajar con comunidades, organizaciones e instituciones que estructuralmente responden a problemas o cuestiones sociales detectadas como sentidas. De los proyectos revisados en este trabajo, hay variedad de destinatarios con quienes se genera el vínculo para desarrollar la intervención. Respecto a la variable del estado inicial asumido, en los proyectos $A+S$ no necesariamente esto es explícito. Por ejemplo, desde la intervención social, si un proyecto pretende asesorar en planes de marketing a microempresarios, es importante que se conozcan las condiciones iniciales de cada uno para entender cuál puede ser la contribución del servicio a desarrollar, pues seguramente no todos los microempresarios llegarán en igualdad de condiciones a la asesoría. Aquí se requiere explícitamente que el modelo $A+S$ avance, desde la detección de necesidades y la comprensión del sentido de desarrollar $A+S$.

John McKnight (1996) discute el concepto de necesidad que a menudo tienen los estudiantes. La necesidad, dice, a menudo se define como una deficiencia o como la falta de algo que un cliente necesita o desea. La deficiencia se coloca en el otro en tanto individuo y no como una condición sistémica. Esta comprensión de la necesidad como deficiencia individual refuerza la comprensión simplista de los problemas sociales e ignora los recursos y fortalezas que ya existen en las comunidades.

De la mano con lo anterior, el tipo de cambio perseguido, desde el punto de vista de la intervención, es una situación bastante desafiante en el aprendizaje-servicio. En parte porque ese estado deseado implica transformaciones complejas de las estructuras o bien de procesos sociales dentro de una comunidad. ¿Será que un proyecto $A+S$ puede alcanzar eso 0 será necesario pensar en varios proyectos? Desde su implementación, quizás sea posible pensar que las características de diseño e implementación tradicional de un curso $A+S$ (habitualmente con un objetivo muy concreto, con recursos limitados y tiempo acotado) sea impensado, pero 
que la concatenación de varios proyectos, sí. Siguiendo con el esquema de intervención, se observa que los objetivos o metas del $A+S$ tienen que ir más allá del objetivo de servicio. Se necesita un objetivo de cambio claro. No se trata de objetivos que hablen de las acciones que harán los estudiantes, como por ejemplo levantar información de puntos críticos de una zona semi rural de la Región Metropolitana o asesorar en planes de marketing a microempresarios pues en esa semántica, el estándar de calidad está puesto en si se cumplió o no la acción determinada, pero nada dice respecto al logro de metas de cambios. ¿De qué sirvió enseñarle un plan de marketing? ¿Mejoró la calidad de vida de los microempresarios? Esta es otra clave de contribución de la perspectiva de la intervención social, delimitar y medir los objetivos planteados.

Los ámbitos de aplicación de la intervención social cubren un amplio escenario de fenómenos sociales, en los cuales el $A+S$ se inscribe desde las particularidades de cada disciplina. Si bien este punto está abordado desde la metodología, donde sí se podría avanzar de manera institucional, es a través de la delimitación de temas prioritarios, en los cuales la UAH ya ha avanzado, definiendo algunos grupos de interés para la Compañía de Jesús (pueblo Mapuche, población migrantes, juventud, marginación, ecología y género).

Respecto a las técnicas usadas, de los proyectos analizados, algunos tenían la posibilidad de utilizar técnicas más personalizadas de intervención que se ajustaban tanto a los requerimientos de la comunidad como a los propósitos de aprendizaje del curso, así como otros tenían un abordaje grupal para entender una problemática y traducirlas en un servicio.

Finalmente, sobre la duración temporal, es un elemento que se presenta como un desafío para el $A+S$, dado que los parámetros de la intervención apuestan a periodos largos entre planificación y dinamización de comunidades y ejecución de acciones, que no calzan con los tiempos académicos de un semestre, que a su vez, se restringe a veces incluso a semanas de trabajo. Esta preocupación corrobora la literatura que ha analizado la importancia del tiempo como factor de aprendizaje para los estudiantes y de calidad del servicio para las comunidades (Eyler, Giles, Dwight y Braxton, 1997; Mabry, 1998; Patterson, 1987, Sandy, 2007).

\section{Conclusiones}

El propósito de este estudio fue analizar los resultados de la evaluación que estudiantes de la UAH hacen sobre el aporte del aprendizaje-servicio tanto para su formación profesional como para las organizaciones sociales. Con estos resultados se buscaba contribuir a mejorar al modelo de implementación, desde el punto de vista teórico y práctico. Es posible afirmar que los estudiantes sí consideran que es un aporte al aprendizaje, ya sea porque pone en valor la relación teoría/práctica, reconocen que en esta metodología pueden acercarse al mundo laboral o porque a través de esta metodología, les permite aprender mejor los contenidos del curso. 
Dependiendo de las características de los proyectos en materia de diseño del servicio, algunos podrían estar aportando más a la solución de las necesidades del socio comunitario. De acuerdo a la perspectiva estudiantil, cuando los proyectos generaron una mayor vinculación con la comunidad, se logró aportar de manera más significativa al socio comunitario. Como esto fue analizado desde la visión del estudiante, es una respuesta parcial y requiere nuevos estudios para corroborar, sobre todo, desde la perspectiva de los socios comunitarios. Finalmente, la información recogida permite reconocer aspectos muy concretos de la implementación más práctica de un curso $A+S$, como mejorar la comunicación con el socio comunitario o equilibrar la carga académica de trabajo.

A partir de los proyectos analizados se puede indicar que los resultados muestran varias dimensiones de aprendizajes para los estudiantes, desde su formación académica disciplinar hasta aprendizajes más actitudinales. Aunque este estudio no permite generalizar estos resultados, sí es posible reconocer que tiene una alta coherencia con la evidencia nacional e internacional sobre los alcances de la metodología. También es posible indicar que, como se ha analizado en extenso, el servicio en $A+S$ es un ámbito a mejorar. Esto implica entender que no basta solo con la elaboración de productos, sino que implica que las comunidades participen en cada etapa de un proyecto $A+S$, desde el diseño, la implementación y evaluación. En ese proceso, la comunidad juega un rol clave. En esa misma línea, la literatura de la intervención social ayuda a entender que no basta solamente con la elaboración de productos. Ofrece un marco de referencia que se constituye en un hallazgo interesante para el modelo $A+S$ de cómo mejorar el rol de los socios comunitarios y la arista del servicio en el $A+S$.

\section{Referencias bibliográficas}

Alfaro, J. (2000). Discusiones en Psicología Comunitaria. Santiago: Universidad Diego Portales.

Aramburuzabala, P., y García, R. (2013). Aprendizaje-Servicio en la formación de maestros comprometidos con la justicia social. En Rubio, L. ; Prats, E. y Gómez, S. (Coord.) (2013). Universidad y sociedad. Experiencias de aprendizaje servicio en la universidad (pp. 14-21). Barcelona: Universitat de Barcelona.

Batlle, R. (2013). El AprendizajeServicio en España. El contagio de una revolución pedagógica necesaria. Barcelona: Editorial PPC.

Berger, P. y Luckmann, T. (1967). La construcción social de la realidad. Buenos Aires: Amorrortu.

Bernasconi, A. y Rojas, F. (2003). Informe sobre la Educación Superior en Chile: 1980-2003. Santiago: Digital Observatory for Higher Education in Latin America and the Caribbean.

Berríos, V., Contreras, A., Herrada, M., Robles, M. y Rubio, X. (2012).

Resultados de Aprendizaje Servicio en la UC desde la mirada de sus actores: 
Docentes, estudiantes y socios comunitarios. Recuperado de: http://desarrollodocente.uc.cl/images/D iscusi\%C3\%B3n Resultados de AS en la UC actores.pd

Bringle, R. y Hatcher, J. (1996). Implementing Service Learning in Higher Education. Journal of Higher Education, 67(2), 221-239.

Butin, D. (2010). Service-Learning in theory and practice: the future of community engagement in higher education. New York: Palgrave Macmillan.

Caire, M. (2015). Cinco buenas prácticas para conformar una sociedad de aprendizaje servicio. RIDAS Revista Iberoamericana de Aprendizaje Servicio, 1, 144-149. Recuperado de: http://revistes.ub.edu/index.php/RIDAS Larticle/view/ridas2015.1.10

Dahan, T. (2016). Revisiting Pedagogical Variations in ServiceLearning and Student Outcomes. International Journal of Research on Service-Learning and Community Engagement, 4(1). Recuperado de: http://journals.sfu.ca/iarslce

Deans, T. (1999). Service-Learning in Two Keys: Paulo Freire's Critical Pedagogy in Relation to John Dewey's Pragmatism. Michigan Journal of Community Service Learning, 6, 15-29.

Donoso, P. (1993). Extensión universitaria: Una aproximación para su análisis. Santiago: Corporación de Promoción Universitaria.

Eyler, J., Giles, J., Dwight, E. y Braxton,
J. (1997). The impact of servicelearning on college students. Michigan journal of community service learning, 4, 5-15.

Eyler, J. y Giles, D. (1999). Where's the learning in service-learning? San Francisco: Jossey-Bass Publishers.

Eyler, J. y Giles, D. (2001). At a glance: what we know about the effects of Service-Learning on College Students, Faculty, Institutions and Communities. Nashville: Vanderbilt University. Corporation for National Service Learn and Serve America National Service Learning Clearinghouse.

Fernández, E.; Ceinos, S. y Nogueira, M. (2017). Relaciones entre el Aprendizaje-Servicio (ApS) y la orientación e inserción profesional de los/las universitarios/as. En AAVV (2017). Aprendizaje-Servicio e innovación en la universidad. VII Congreso Nacional y II Internacional de Aprendizaje-Servicio Universitario. Santiago: Universidad de Santiago. pp. 225-232.

Folgueiras, P.; Luna, E. y Puig, G. (2013). Aprendizaje y servicio: estudio del grado de satisfacción de estudiantes universitarios. Revista de Educación, 362, 159-185.

Furco, A. y Billig, S. (2002). ServiceLearning: the essence of the pedagogy. Nashville: National Service-Learning Clearinghouse.

Furco, A. (2003). El impacto educacional del aprendizaje-servicio. ¿Qué sabemos a partir de la investigación? California: University of 
California-Berkeley. Recuperado de https://roserbatlle.files.wordpress.com/ 2009/02/resultats-investigacio-apsfurco-modo-de-compatibilidad.pdf

Furco, A. y Root, S. (2010) Research desmonstrates the value of ServiceLearning. Phi Delta Kappan

International.

Jouannet, C.; Salas, M. H. y Contreras, M. A. (2013). Modelo de implementación de Aprendizaje Servicio $(A+S)$ en la UC: Una experiencia que impacta positivamente en la formación profesional integral. Calidad en la Educación, 39, 197-212. Recuperado de https://scielo.conicyt.cl/scielo.php?scrip $\mathrm{t}=\mathrm{sci}$ arttext\&pid $=\mathrm{S} 0718$ $\underline{45652013000200007}$

Kolb, D. (1993). The Process of Experiential Learning. En Thorpe, M.; Edwards, R. y Hanson, A. (eds) (1993). Culture and processes of adult learning pp.138-156, London: Routledge.

Krause, M. y Jaramillo, A. (ed.) (1998). Intervenciones PsicológicoComunitarias en Santiago de Chile. Santiago: Pontificia Universidad Católica de Chile.

Lapalma, A. (2001). El escenario de la intervención comunitaria. Revista de Psicología de Universidad de Chile, $\mathrm{X}(2)$.

Mabry, B. (1998). Pedagogical variations in service-learning and student outcomes: How time, contact, and reflection matter. Michigan journal of community service learning, 5, 3247.
Martinez, V. (2007). Modelos operativos, modelos de acción y comunidades de prácticas en los programas de intervención social. En AAVV (2007). Trayectorias de la Psicología Comunitaria en Chile. Prácticas y Conceptos. Valparaiso: Universidad de Valparaíso. pp. 187-214.

McKnight, J. (1996). The careless society: Community and its counterfeits. New York:Basic Books.

Ochoa, E. (2010). Aprendizaje-Servicio en América Latina: apuntes sobre pasado y presente. En AAVV (2010). Tzhoecoen, Revista Científica. Universidad Señor de Sipán, 3(5), 108125.

Opazo, H. (2015). Experiencias de aprendizaje-servicio en la formación del profesorado. Un estudio de caso. Tesis doctoral. Universidad Autónoma de Madrid.

Patterson, E. (1987). Effects of participation in required and not required community service programs on the process of self actualization in high school students. Doctoral dissertation, University of Florida.

Saavedra, C. (2005). Aproximación al desarrollo actual de la Psicología Comunitaria desde el análisis de las prácticas que ésta construye en el campo de la intervención social. Tesis de Magíster Psicología Comunitaria, Universidad de Chile.

Sánchez, A. (1986). Intervención comunitaria: introducción conceptual, proceso y panorámica. En A. Martín González, F. Chacón Fuertes y M. 
Martínez García (Eds). Psicología Comunitaria (pp. 169-186) Madrid: Visor.

Sánchez, A. (1991). Psicología comunitaria. Bases conceptuales y operativas. Métodos de intervención. Barcelona: PPU.

Sandy, M. (2007) Community Voices: A California Campus Compact Study on Partnerships. Final Report. Higher Education. Paper 143. Recuperado de http://digitalcommons.unomaha.edu/sl cehighered/143

Schmidt, A. y Robby, M. (2002). What's the value of service-learning to the community? Michigan Journal of Community Service Learning, 9(1), 2733.

Stanton, T. (1990). Service learning and leadership development: Learning to be effective while learning what to be effective about. In Service-Learning and Leadership Development.Raleigh, NC: National Society for Internships and Experiential Education, pp. 336-352.

Tapia, M. (2006). Aprendizaje y servicio solidario. Buenos Aires: Editorial Ciudad Nueva.

Tapia, M. (2008). Aprendizaje Servicio en la Educación Superior. Una mirada analítica desde los protagonistas.

Buenos Aires: Ministerio de Educación Presidencia de la Nación, Argentina.

Tryon, E. y Stoecker, R. (2008). The Unheard Voices: Community Organizations and Service-Learning. Journal of Higher Education Outreach and Engagement, 12(3).
UNESCO (1998) La educación superior en el siglo XXI, visión y acción. Conferencia mundial de Educación Superior. Paris:Unesco.

Universidad Alberto Hurtado (2008) Proyecto Formativo. Recuperado en: http://www.uahurtado.cl/pdf/Proyecto Formativo UAH.pdf 